\section{NGOZI ONWURAH Y PRATIBHA PARMAR: DE ÁFRICA A EUROPA}

\author{
Alejandra Moreno Álvarez \\ Universidad de Oviedo \\ morenoalejandra@uniovi.es
}

\section{NGOZI ONWURAH AND PRATIBHA PARMAR: FROM AFRICA TO EUROPE}

\begin{abstract}
RESUMEN: La directora nigeriana Ngozi Onwurah y la keniata Pratibha Parmar utilizan la gran pantalla para deconstruir el mito creado por occidente en torno a las mujeres africanas. Lo hacen desde la diáspora, ya que ambas viven y producen su trabajo en Inglaterra, plasmando en sus documentales, cortos y largometrajes el difícil y largo proceso de convivialidad no ya interracial sino también sexual en el Reino Unido. Onwurah y Parmar hacen uso de sus cámaras para denunciar el acoso sufrido por ambas tanto por su color de piel como por, en el caso de Parmar, su homosexualidad. Ambas coinciden en señalar el reto que les presenta obtener subvenciones, ya que debido a la temática de sus trabajos la audiencia se supone minoritaria. Su propósito no es solo llegar a las minorías, sino que su mensaje sea visto y escuchado por el público en general.
\end{abstract}

PALABRAS CLAVE: Colonialismo, convivialidad, diáspora, exótico, homosexualidad, racismo.

\section{INTRODUCCIÓN}

La directora nigeriana Ngozi Onwurah y la directora asiático-británica Pratibha Parmar han desarrollado, al mismo tiempo que lo han plasmado en sus trabajos, un sentido de la identidad híbrido al no estar unidas a un solo lugar geográfico y dado que diferentes espacios se han convertido en sus puntos de referencia. Las artes visuales son para Onwurah y Parmar espacios naturales donde los sujetos nómadas se convierten en una entidad que se redefine para ser identificada, siendo este uno de los objetivos de sus obras. Ambas coinciden en señalar lo ya articulado por Lan Dong, "Migrants have to ask themselves questions about where they are before they can even begin to solve the problem related to who they are" (Dong, 2004, 66), y también que los nativos se han de hacer la misma pregunta, ya que su pais natal ha sido alterado por la pluralidad y diversidad que aportan los sujetos que lo habitan hoy en día. Tanto Onwurah como Parmar trazan, en varios de sus documentales, la historia del colonialismo británico, dado el gran impacto que ha tenido en sus familias. Onwurah nace en Nigeria, de padre

\begin{abstract}
Nigerian director, Ngozi Onwurah, and Kenyan director, Pratibah Parmar use the big screen to deconstruct the myth of African women in Western culture. Both of them work and live in the diaspora, in Great Britain, and it is there that they denounce, in their documentaries, short and feature-length films, the arduous process people go through to achieve a peaceful coexistence despite differences of sexuality as well as race. Onwurah and Parmar use their cameras to expose the abuse they both suffered, and still do, on account of the colour of their skin and, in the case of Parmar, her homosexuality. Both directors agree on the challenge that obtaining funding represents, since their work is considered to be aimed at a minority audience. However, their goal is to reach not just those minorities but the public at large.
\end{abstract}

KEY WORDS: Colonialism, conviviality, diaspora, exotic, homosexuality, racism.

nigeriano y madre blanca y británica. Tanto ella como su madre y hermano huyen de Nigeria escapando así de la Guerra Civil. En el caso de Parmar, su madre nace en Kenia, es luego educada en la India, donde se casa, y posteriormente regresa a Kenia. Más tarde es forzada a dejar el país y a construir una nueva vida en Inglaterra. Este bagaje familiar influye en la forma de pensar de ambas directoras, que tratan de responder en sus trabajos a cuestiones de identidad y pertenencia.

Onwurah y Parmar parecen coincidir con la postura del crítico Paul Gilroy, quien considera, en After Empire: Melancholia or Convivial Culture? (2004), que el término ideal para definir "the processes of cohabitation and interaction that have made multiculture an ordinary feature of social life in Britain's urban areas and in postcolonial cities elsewhere" es el de convivialidad (Gilroy, 2004, xi). Para Gilroy, la convivialidad no elimina el racismo, ni inscribe el triunfo de la tolerancia, pero sí sugiere un espacio diferente para los rituales que han alterado su significado ante la ausencia de creencias exacerbadas en una raza absoluta (Gilroy, 2005, xv). Es decir, la con- 
vivialidad, que tanto Onwurah como Parmar aspiran a plasmar en sus obras, es el resultado inevitable de décadas de cohabitación de diferentes razas en Gran Bretaña; se trata de una convivialidad espontánea y no impuesta. Así pues, este artículo pretende hacer un repaso de la obra de Onwurah y Parmar, no tanto por el hecho de que ambas hayan nacido en África y vivan, por determinadas razones, en el Reino Unido, sino porque sus discursos visuales de denuncia instan al pluralismo con el propósito de que las identidades hibridas se muevan hacia la convivialidad aquí señalada. Es el propio Gilroy quien enfatiza la necesidad de que las artes contribuyan a este proceso (Gilroy, 2004, 157). El artículo aborda dos aspectos fundamentales en la filmografía de estas autoras. Uno de esos aspectos es la otredad de los sujetos no europeos, tema este perfectamente trazado por Onwurah en sus obras, una directora para quien "[t]he body is the central landscape of an anti-imperialist cinematic discourse" (Foster, 1997, 24). El otro aspecto destacado es abordado en la obra de Parmar, que ofrece un discurso que va más allá de la convivialidad racial para añadir la sexual.

\section{Ngozi Onwurah: Convivialidad racial}

Ngozi Onwurah nace en Nigeria, hija de un nigeriano de color y de una madre escocesa blanca. Ella y su hermano, Simon Onwurah, pasan parte de su niñez en Nigeria hasta que esta entra en guerra. La madre de Onwurah huye con sus dos hijos a Inglaterra mientras que su padre se queda para tomar parte en la guerra. Sin embargo, la seguridad que supuestamente iban a hallar en Inglaterra llevaba consigo un precio. Si bien se habían ido de Nigeria a causa de la guerra en Biafra, una vez en Inglaterra se dieron cuenta de que habían aterrizado en otra zona de guerra y que solamente ellos eran conscientes de esto. Onwurah y su hermano eran negros y su madre blanca. La familia se aloja en un barrio de Newcastle, área hasta entonces no habitada por población de color. Onwurah y su hermano son enseguida objeto de abusos. No solamente reciben insultos, sino que también les ponen excrementos a la puerta de casa, les hacen meter sus cabezas en los váteres, etc. Ambos están tan desesperados por ser aceptados que llegan incluso a bañarse en lejía con la ilusión de aclarar la tonalidad de su piel. Onwurah da cuenta de todo esto en su documental Coffee Coloured Children (1988).
Ya de adolescente y cansada de los abusos de los que asiduamente era objeto, Onwurah deja su pasividad a un lado para convertirse en un sujeto activo. Cumplidos los quince años, y harta de su estilo de vida, se va de casa con el consentimiento de su madre. Será entonces, en un tren camino de Londres, cuando una representante de modelos se dirija a ella atraída por la belleza exótica de Onwurah. Esto la llevó, en los años ochenta, a una exitosa carrera como modelo en Londres. Al mismo tiempo, Onwurah compagina su carrera de modelo con sus estudios de cine en St. Martin's School of Arty The National Film and Television School, ambas en Inglaterra. Tras tres años de modelo y cansada de llevar una dieta a base de pastillas y de abstinencia, decide volcarse en su carrera como directora. Onwurah dirige varios cortos, documentales, capítulos de series de televisión y filmes. Welcome /l the Terrordome (1994) es su primer largometraje; gracias a él se convierte en la primer mujer negra que dirige una película de estas características en el Reino Unido.

\subsection{Documentales}

Onwurah completa en 1988 su primer trabajo como directora con el documental Coffee Colored Children. El corto recibe buenas criticas y es premiado en el British Broadcasting Corporation (BBC), el National Black Programming Consortium y el San Francisco Film Festival. El documental, de quince minutos de duración, recoge las experiencias de dos hermanos negros que crecen bajo la sombra del racismo. La película es para Onwurah una especie de exorcismo que le permite cuestionar la celebración del llamado melting pot (Foster, 1997, 27). El documental, de narración poética, consagra el estilo de Onwurah, que es definido como magistral y que muestra la cruda realidad al mismo tiempo que provoca emociones en la audiencia. La directora combina aquí fantasía y realidad, y se basa en las experiencias vividas y sufridas por ella misma. Las mismas experiencias que en la adolescencia la llevan a utilizar la fuerza bruta la conducen, ya como directora, a plasmarlas en la pantalla y así articular lo que es difícil explicar con palabras.

En los años noventa produce una serie de trabajos provocadores, como los documentales Fruits of Fear (1990) o Who Stole the Soul? (1992). Este último recibe el premio RTS Television Award y el Best Adult Continuing de la Royal Television Society. Onwurah examina cómo el poder blanco 
se ha apropiado de la música negra. Le sigue, en 1993, el documental And Still I Rise, que escribe y dirige. Esta adaptación del poema de Maya Angelou explora los estereotipos que rodean a las mujeres negras. Onwurah insta a estas mujeres a defender su propia sexualidad y pluralidad de identidades, y denuncia que el rechazo que en ocasiones reciben es producto de la necesidad que mucha gente tiene de la otredad para así ellas/os poder definirse. Como dice Ferguson, "[i]t would seem that Orientalism in film and television is virtually synonymous with a generic form of melodrama where any imperialist or post-imperialist can celebrate their power (or lost power) through their representation of the Other" (Ferguson, 1998, 71).

En ese mismo año dirige Monday's Girls (1993), documental que cuestiona las tradiciones culturales africanas en un mundo moderno definido por la individualidad. Cuenta la historia de dos jóvenes mujeres nigerianas que son invitadas a la tradicional ceremonia de iniciación de las mujeres. Una de ellas, Florence, lo considera un honor, mientras que la otra, Asikiye, occidentalizada, piensa que dicho evento está pasado de moda y atenta contra la dignidad de las mujeres. Onwurah narra cómo ambas mujeres son encerradas durante cinco semanas en unas fattening rooms (Foster, 1997, 35), después de lo cual salen para celebrar que son mujeres de la comunidad. Onwurah trata en todo momento de no caer en la yuxtaposición binaria occidental que tiende a representar esta ceremonia como sexista y salvaje. Así, las mujeres hablan tanto de lo divertida como aburrida que resulta esta ceremonia. Florence considera que gracias al ritual se ha convertido en una mujer de verdad (Foster, 1997, 35), en una mujer de la comunidad; mientras que Asikiye no soporta siquiera ver sus pechos desnudos, y es que su occidentalización se lo impide. Monday's Girls fuerza el debate sobre las interpretaciones conflictivas resultado de ideologías culturales y sentidos de espacio definidos culturalmente, lo que Hall define como "the West and the Rest" (Hall, 1992). Onwurah celebra que estas mujeres iriabo (vírgenes), tienen en estas "habitaciones de engorde" un espacio propio al mismo tiempo que desconocido por el mundo occidental.

En 1995 dirige The Desired Number, donde cuenta que las familias de Iwollo, en Nigeria, tienen al menos nueve niños dada la necesidad de mano de obra y la alta mortalidad en el país. Onwurah denuncia que occidente, en lugar de reforzar las políticas de control de natalidad en Nigeria, debería mantenerse al margen y evitar enviar a Nigeria misioneros cristianos que sermonean contra las prácticas de control de natalidad. Onwurah deconstruye, tal y como hemos comentado, el mito que occidente tiene de las mujeres africanas y al mismo tiempo inscribe otro porqué de las cosas, dando así a conocer a "the West", "the Rest".

\subsection{Cortos}

El corto más aclamado de Onwurah, y uno de los más comercializados en el Reino Unido, es The Body Beautiful (1991), galardonado con el premio al mejor cortometraje en el Melbourne International Film Festival y en el Montreal Film Festival. Este trabajo autobiográfico explora la relación entre Onwurah y su madre, Madge. El corto trata de dos historias paralelas: la historia de una madre que se casa con un nigeriano, cría a dos hijos de raza mixta y posteriormente se enfrenta a un cáncer de pecho y a una mastectomía. Por otro lado, la historia de una hija que crece con una visión diferente de la de su madre blanca, que es objeto de mirada por su otredad, y que admite ser incapaz de ver a su madre como un ser sexuado. El trabajo se remonta así a los años en los que Onwurah era modelo y, mientras esta está inmersa en su carrera, donde el cuerpo lo es todo, su madre experimenta la decadencia de su propio cuerpo tras la mastectomía. Onwurah analiza las políticas corpóreas del momento, donde el peso y la imagen del cuerpo llegan a afectar a muchas mujeres, y también explora las relaciones madrehija. Con este corto Onwurah proporciona un espacio a su madre, como protagonista de la historia y como actriz, dado que la propia madre de Onwurah se interpreta a sí misma. En una de las escenas más impactantes madre e hija aparecen en una sauna pública; la madre se quita la toalla, exponiendo la cicatriz resultante de la mastectomía, lo que provoca rechazo en las mujeres que en ese momento comparten espacio con ella. Onwurah, como directora, ve a su madre a través de la cámara, lo que hace que acepte el nuevo cuerpo de su madre tras la mastectomía, y también la observa a través de los ojos de la actriz que interpreta su papel de hija, viendo así Onwurah a su madre como madre, como mujer, como ser sexuado. Al igual que Onwurah no puede experimentar la mastectomía de su madre, nosotras/os, como espectadoras/es, no podemos entender lo que significa crecer en el Reino Unido como mujer de color y de madre blanca (Foster, 1997, 33). 


\subsection{Filmes}

Con la película Welcome II the Terrordome (1994), que recibe el premio del público en el Verona Love Screens Film Festival (1995), Onwurah se consagra como una contadora de historias de renombre. Su estreno es sonado, dado que se trata del primer largometraje dirigido por una mujer de color en el Reino Unido. Fiel a su estilo, la película recoge temas como el racismo e incluye amor interracial y odio racial. Las criticas alaban a esta directora por ofrecer al público un testimonio de la convivialidad tan desgarrador como honesto. En las producciones hollywoodienses las mujeres y hombres negros son devaluados constantemente, de ahí que Onwurah cree una ciudad distópica y futurista donde solamente vive la población negra y donde su sustento económico sea la venta de drogas. Como señala Ferguson, "[t]here is much to substantiate this line of reasoning in the representations of issues of 'race' which abound in the mass media. These would include the ways in which popular histories are constructed, the numerous adventure stories which tell of daring deeds in distant places (distant from Europe or the USA)" (Ferguson, 1998, 69). Dos bandas formadas por gente de color se pelean mientras hacen negocios con clientes blancos. Angela, casada con Black Rad, intenta proteger a su hijo Hector del mundo de las drogas. El hermano de Black Rad, Spike, vive con una mujer blanca, Jodie, que está embarazada. El exnovio blanco de Jodie, Jason, es racista, y cuando ve a Jodie con Spike denuncia a la banda de Spike ante la policía. Jason asalta a Jodie y la golpea hasta que le provoca un aborto. Mientras tanto, Hector muere cuando la policía está arrestando a la banda de Spike. Como consecuencia de esto, Angela mata a Jason y al policía blanco que intenta arrestarla. La familia de Angela culpa a Jason y a Jodie de la muerte de Hector y del arresto de Angela. La abuela de Hector, Rosa, culpa a los blancos de todo este odio, mientras una voz en off de un hombre negro explica que solamente siente furia. Entre tanto, se escuchan extractos de discursos de Malcom X, "Until the American negro lets the White man know that we are really really ready and willing to pay the Price that is necessary for freedom, our people will always be walking around here second-class citizens, or what you call, 'twentieth century slaves.' The price of freedom is death" (Foster, 1997, 39).

Onwurah recontextualiza la opresión de hombres y mujeres de color por parte de la población blanca y, en este caso, el papel que tuvieron las mujeres blancas. De ahí que las primeras escenas de la película se remonten a una playa de Carolina del Sur en 1652, donde varios africanos son "vendidos" ante la expectación del gentío blanco. Con el fin de evitar acabar sus días como esclavos, varios africanos huyen sumergiéndose en el océano para, "metafóricamente", volver a "casa". Jodie, representada en el pasado, intercambia una mirada con un hombre negro; de esta forma, Onwurah muestra el hecho de que la mujer blanca ve al hombre negro como exótico. Combinando pasado, presente y futuro, la directora se acerca con su discurso cinematográfico a lo hiperreal e inscribe un nuevo espacio donde los cuerpos de la población negra flotan a través del tiempo y del espacio, trasgrediendo las estructuras binarias hasta ahora establecidas por el discurso dominante. $Y$ de ahí viene el título, tomado del grupo de rap Public Enemy, ya que dome significa cúpula, "la cúpula del terror". El discurso dominante ocupa la cúpula de la pirámide; ahí se construyen los significados y los sujetos activos crean a los pasivos. Esta cúpula es el panóptico de Foucault, espacio en el que los blancos venden la droga a los negros, encasillándolos así en un gueto de donde es tarea ardua salir. Pero Onwurah deconstruye ese panóptico y hace que los negros se empoderen ocupando un lugar activo en la cúpula. De ahí la bienvenida al nuevo panóptico "Welcome II". Para ellos la muerte, representada violentamente en la pantalla, no es el final, sino el inicio del viaje espiritual (Foster, 1997, 41).

\subsection{Otros}

Onwurah es también promotora del cine africano. En 2001 trabaja en un proyecto denominado Mama Africa que trata de dar a conocer el talento de directoras de cine africanas y al mismo tiempo deconstruye los estereotipos occidentales sobre las mujeres contemporáneas africanas. El proyecto consta de seis cortos, uno de ellos es Hang Time. El corto, dirigido por Onwurah, trata de un adolescente nigeriano y de su deseo de ser jugador en la NBA. Onwurah describe el sueño americano desde la perspectiva de un africano. En el transcurso hacia esta ambición el africano se ve envuelto en un crimen, vende su alma y aprende lo que significa la lealtad a la familia. Onwurah trata aquí, en todo momento, de aunar África con las experiencias afroamericanas. En el San Diego Union and Tribune (24 de enero, 1999), Onwurah señala que el cine africano es difícil de comercializar y, si quieren prosperar y darlo a conocer, el mercado está en una 
audiencia afroamericana (citado en "Afrocentric News"). Teniendo en cuenta el impresionante trabajo que hasta ahora ha realizado, Onwurah tiene la capacidad de llevar el cine africano no solo a la audiencia afroamericana, sino también a la norteamericana de diferentes razas y culturas. Si bien su trabajo trata principalmente de sujetos que luchan por encontrase a sí mismos, los espectadores y las espectadoras, independientemente del color de su piel, se verán identificados/as.

\section{Pratibha Parmar: Convivialidad sexual}

Pratibha Parmar nace en Nairobi, Kenia, de ascendencia India. En 1967, y siendo una niña, su familia se traslada a Inglaterra. Estudia en la Universidad de Bradford, donde se gradúa y realiza sus estudios de postgrado en el centro de estudios culturales de la Universidad de Birmingham. Comienza su carrera cinematográfica con la realización de documentales que ya esbozan la pasión de Parmar, que no es otra que la de dar vida en la pantalla a nuevos sujetos que poco tienen que ver con los creados por el discurso dominante. Parmar crea así un sujeto hibrido que, como definen Shohat y Stam, es "dynamic and mobile and is constituted through an 'unstable constellation of discourses'" (Shohat y Stam, 1994, 42). Su trabajo, por el que ha recibido numerosos premios, se centra en la representación de mujeres, siempre abordando temas sociales. En octubre de 2007 es premiada con el Visionary Award por su obra presentada en el One in Ten Film Festival. También obtiene el Life Time Achievement Award en el San Francisco Frameline Film Festival. En su producción destacan los vídeos musicales para artistas de renombre como Morcheeba, Tori Amos, Ghostlands y Midge Ure. También es una reconocida escritora; entre sus obras cabe señalar Warrior Marks: Female Genital Mutilation and the Sexual Blinding of Women (1996), libro escrito conjuntamente con Alice Walker, y la edición de Charting the Journey: Writings by Black and Third World Women (Grewal et al., 1988).

Las películas de esta directora polifacética, que cuenta con su propia productora (Kali Films), han sido exhibidas en festivales internacionales. Dada la temática que aborda en sus trabajos es, al igual que Onwurah, objeto de estudio en los departamentos de Estudios de la Mujer o Estudios Cinematográficos, de ahí que sea invitada a impartir char- las en universidades como la Florida Atlantic University o la University of California. Imparte talleres en el Actor's Centre de Londres. Actualmente, Parmar tiene entre sus futuros proyectos realizar un documental sobre Drag Queens y también dirigir una película. Este último proyecto trata el tema de cómo la mayoría de las mujeres del mundo no se siente conforme con el cuerpo con el que han nacido y cómo esta experiencia lleva, con frecuencia, a mujeres de diversas culturas a modificar, reformar y alterar sus cuerpos para "encajar" en los cánones estéticos establecidos. Para este proyecto trabaja junto a Eve Ansler, autora de The Vagina Monologues.

\subsection{Filmes}

En su filmografía destaca, como ya hemos apuntado, la temática de sus cortos, documentales y filmes. Su obra más reciente es el filme que dirige en 2006 y que lleva por título Nina's Heavenly Delights. Se trata de una pelicula ambientada en Glasgow que narra la historia de una mujer de ascendencia India que regresa a Escocia para el funeral de su padre. Alli descubre que su progenitor, dueño de un restaurante especializado en curry, había solicitado concursar en la Best of the West Curry Competition. La protagonista decide retornar a Glasgow para hacerse cargo del restaurante de su padre. A través de la comida, Parmar nos habla del amor, de los matrimonios de conveniencia y de la dificultad de la convivialidad en Glasgow. Entre receta y receta, y con la banda sonora de Daydream Believer de The Monkees, el espectador se olvida de los puntos principales que aborda Parmar, como la relación homosexual de la protagonista, para sumergirse en esta película de blockbuster con tintes de Bollywood. Ya en las primeras escenas, Lisa, interpretada por Laura Fraser, llega al funeral de su padre en una furgoneta que tiene un eslogan pintado que dice "Live your dream". Contrasta así la muerte con la sugerencia de vivir un sueño, lo cual acaban consiguiendo los protagonistas de la película, puesto que Lisa logrará llevarse el premio al restaurante con el mejor curry y también sellará con un beso ante las cámaras su relación lésbica con Nina, interpretada por Shelley Conn. El hermano de Lisa reconoce ante su madre que se ha casado con una chica blanca escocesa y la propia madre admite que quiso al padre de sus hijos, pero que de quien está verdaderamente enamorada es del dueño del restaurante "The Jewel of the Crown". Termina el filme con un baile escocés interpretado por la hermana pequeña de Lisa. Así, 
esta película, que se asemeja a Como agua para chocolate (1992) del director mejicano Alfonso Arau y basada en la novela del mismo título de Laura Esquivel, une a Escocia y a India, siempre representada en la película por el curry y el monumento emblemático del Taj Mahal, y muestra el estado de convivialidad racial y sexual articulado por Gilroy. Parmar nos recuerda, como también hacen Shohat y Stam, que "Europe itself is a synthesis of many cultures, and that the West is a 'collective heritage, an omnivorous mélange of cultures'" (Shohat y Stam, 1994, 14).

\subsection{Televisión y cortos}

Parmar admite la dificultad de encontrar financiación para producir Nina's Heavenly Delight, puesto que no solamente aborda la convivialidad interracial sino también el lesbianismo. No es de extrañar, por tanto, que esta directora, para mantener su productora, dirija series para la televisión de carácter comercial, como varios capitulos para la serie Doctors (2002): Trapped; Never Forget; Carry That Weight; Golden Years y A Matter of Perspective. Al igual que Onwurah, también dirige varios capítulos para la serie de televisión Siren Spirits (1995). No obstante, al igual que hace en su único largo, trata de introducir en sus trabajos la temática social, como es el caso de los cortos Playing Dead (2008), Sita Gita (2000) o Wavelenghts (1997), que también tratan del amor. Este último es un cortometraje ambientado en bares gays y donde una mujer intenta encontrar buscar sexo seguro. Memsahib Rita (1994) sigue la misma línea que el corto White Men Are Cracking Up dirigido por Onwurah. Parmar utiliza aquí el realismo mágico y analiza la violencia física y emocional que produce el racismo.

\subsection{Documentales}

Parmar dirige documentales de temática variada, y es en ellos donde profundiza su compromiso social, el mismo que ya de adolescente le llevó a realizar trabajos sociales voluntarios en centros de acogida de mujeres asiáticas. Haremos aquí una selección de los mismos que ordenaremos no de forma cronológica, sino por su temática: racismo, colonización, feminismo y lesbianismo.

Entre los documentales en los que analiza el racismo destaca Warrior Marks (1993). Con esta obra Parmar obtiene el premio otorgado por el público en el 11 Festival Internacional de las Mujeres en Madrid. Para este documental cuenta con la colaboración de Alice Walker, autora, entre otros textos, de El Color Púrpura (1982), premio Pulitzer en 1983. Warrior Marks trata abiertamente sobre la ablación, tema por aquel entonces tabú para la comunidad internacional y que para Parmar, cuyos trabajos abarcan temáticas e ideas que frecuentemente provienen de sus propias pasiones y compromisos, representa un asunto de derechos humanos. Las mujeres de las que se ocupa en este documental no son presentadas como víctimas, sino como mujeres resistentes, luchadoras y que han logrado sobrevivir, y continúan sobreviviendo, a pesar de lo que se les ha hecho. Así pues, Warrior Marks articula la forma de vivir de las mujeres que han sufrido la ablación. La propia Parmar comenta:

Recuerdo haber tenido una larga conversación con Awa Thiam, una feminista africana que escribió un libro extremadamente importante, publicado en el Reino Unido llamado Black Sisters Speak Out (Hablan las hermanas negras) (1978), un análisis feminista sobre la opresión de las mujeres africanas. Awa escribió en su libro un capítulo sobre la mutilación del clitoris y la circuncisión femenina y por haber hablado $\tan$ franca y abiertamente, fue condenada al ostracismo en Senegal. La gente la criticó masivamente, cuestionándola: "¿Por qué expones nuestra cultura de esta manera negativa?" Recuerdo haberle preguntado a Awa, cuando la conoci, en Senegal: "¿Cómo te sientes respecto a que nosotros vengamos acá y hagamos esta película?" Ella dijo: "Sabes, trabajo sobre la base y el convencimiento de que hay una hermandad femenina universal y que estamos todas juntas en esto. Ustedes en Occidente quizás estén luchando contra otras cosas, pero para nosotras la lucha contra la mutilación genital femenina es una prioridad. Ustedes pueden ayudarnos y unirse a nosotras en esta lucha dándole difusión al tema, sacándolo afuera y poniéndolo en la agenda mundial, en el mundo". (Parmar en "Escuela Abierta de Feminismo. Awa Thiam")

Parmar tiene en su agenda denunciar el racismo y "normalizar" la homosexualidad, como ya hemos visto en su filme Nina's Heavinly Delight. El documental Double the Trouble, Twice the Fun (1992) trata sobre la discapacidad y homosexualidad de hombres y mujeres. Fiel a su estilo, la película persigue "normalizar" y no generar sentimientos de compasión en el público. Parmar recrea la dificultad de ser discapacitado y gay, y deconstruye la idea de que la gente discapacitada sea infeliz y no tenga vida sexual. Siguiendo el mismo hilo temático, Khush (1991), premiada 
por el público en el 7 Festival Internacional de las Mujeres en Madrid, recoge la dificultad de ser gay y de color en espacios como Reino Unido, Norteamérica e India. Las barreras geográficas se rompen en esta película para unir experiencias de soledad y, al mismo tiempo, Parmar subraya la sororidad de ser "khush", término que en urdu significa "placer estático".

Esta directora trata también el tema del racismo en Sari Red (1988), documental en memoria de Kalbinder Kaur Hayre, una mujer indo-británica asesinada en 1985 en un ataque racista en Inglaterra. Parmar examina el temor de las mujeres asiáticas ante la violencia tanto en los espacios públicos como privados; de ahí el título de este documental, que se refiere al rojo de la sangre y del sari, y simboliza así la sensualidad e intimidad de las mujeres asiáticas. Dado que ha habido tantos asesinatos racistas en Inglaterra, estos, dice Parmar, a veces se convierten simplemente en estadisticas: "Quise hacer un video que rescatara a esta mujer valiente de convertirse en una nueva estadistica y recordar su vida, sus sueños, su potencial" (Parmar en "Escuela Abierta de Feminismo. Pratibha Parmar"). Este potencial al que hace referencia Parmar es proyectado en el documental A Place of Rage (1991), película sobre mujeres afroamericanas implicadas en el movimiento de los derechos sociales. El documental recoge las entrevistas de Angela Davis, June Jordan y Alice Walter, donde debaten sobre cómo Rosa Parks y Fannie Lou Hamer, entre otras, revolucionaron la sociedad americana.

La temática de la colonización es abordada por Parmar en Bhangra Jig (1990), un corto que realiza por encargo de Channel 4 en 1990 con motivo de la celebración de Glasgow como capital europea de la cultura. Este documental, ambientado una vez más en Glasgow, trata de una mujer asiática que camina por la ciudad observando los iconos arquitectónicos, signos del imperio británico. En contraposición, también observa cómo las comunidades asiáticas han erigido sus propios iconos, reafirmándose no como colonizados, sino ya como habitantes de una ciudad multicultural. El corto ofrece nuevos significados de lo que simboliza ser asiático, británico y europeo. Le precede Brimful of Asia (1988), donde explora la segunda generación de surasiáticos en la cultura británica de finales de los noventa. The Colour of Britain (1994) recoge el trabajo de artistas asiático-británicas/os como Anish Kapoor, Jatinder Verma, Shobhana Jeyasingh, Sutapa Biswas, Chila Kumari
Burman y Zarina Bhimji. Y siguiendo la misma línea, en Emergence (1986) analiza la identidad, alienación y la historia de las mujeres en el contexto de la diáspora. Cuatro mujeres artistas negras y del tercer mundo hablan de sus proyectos, entre ellas la poeta feminista afroamericana Audre Lorde y la artista palestina Mona Hatoum.

Parmar deja clara su militancia feminista en documentales como The Righteous Babes (1998), donde explora la intersección del feminismo con la música popular. La keniatabritánica analiza el papel de las mujeres artistas en los años noventa y su influencia en el mundo moderno, de ahí que estudie figuras destacadas del panorama musical como por ejemplo la rockera Ani DiFranco. Para Parmar la cultura no es monolítica, sino que está en constante movimiento, lo que explica que le guste estar a tono con los cambios y transformaciones; The Righteous Babes es un claro ejemplo. La propia historia de Parmar como cineasta, que en la última década se ha centrado en asuntos con relevancia social y política, particularmente aquellos que afectan a las mujeres, así como su continuo compromiso con el feminismo, ayudaron a dar forma, contenido y estilo visual a este documental. Parmar hace accesible las ideologias feministas a una audiencia más amplia; y es que The Righteous Babes nos recuerda que antes de las Spice Girls existió una larga historia de mujeres en la música, mujeres verdaderamente subversivas y desafiantes.

En cuanto a la homosexualidad, conviene destacar que si ya en Nina's Heavenly Delight Parmar "normalizaba" la sexualidad, lo mismo hace en Jodie: An Icon (1996), documental que ofrece una mirada sobre las maneras en que la actriz de Hollywood Jodie Foster es erigida como icono lésbico. Y lo mismo sucede con Flesh and Paper (1990), documental que resulta ser una exploración lírica de sentidos y sensibilidades de una poeta lesbiana india, Suniti Namjoshi.

\section{Conclusión}

Onwurah y Parmar son grandes narradoras de historias que utilizan la imagen en lugar de la palabra para plasmar la convivialidad contemporánea y los avatares de las minorías. De hecho, Onwurah considera que las africanas y los africanos son estupendos contadores de relatos, dado que así 
es como han conservado su historia. La directora cree que si regresara a Nigeria, un tío suyo se sentaría a su lado y le contaría un cuento que la Ilevaría a 1600, a sus antepasados. De ahí que ella misma se defina como una contadora y directora de historias. No obstante, esta labor no siempre es fácil. El mundo del cine independiente es muy competitivo y depende de las subvenciones. Incluso Onwurah se ha encontrado con situaciones en las que sus películas son subvencionadas con menos presupuesto que las de otras/os directoras/es. En una entrevista para The Independent (25 de noviembre, 1996) Onwurah comenta esta frustración y señala que no entiende por qué la gente piensa que las películas dirigidas por gente de color son más baratas (citado en "Answers.com. Ngozi Onwurah"). Así, Onwurah hace una crítica a la industria del cine británico por relegar, en su programación televisiva, el trabajo dirigido por y para un público de color a una franja horaria de menor audiencia. También se opone a que se considere que el concepto de trabajos dirigidos por gente de color sea destinado a gente de color, dado que muchas historias de negros, especialmente las historias de británicos de color, afectan y tienen que ver también con la gente blanca. Parmar sigue esta misma línea y subraya que "leemos en las imágenes lo que queremos leer en ellas, dependiendo de nuestros propios deseos y necesidades" (Parmar en "Escuelta Abierta de Feminismo. Pratibha Parmar"). Tanto Onwurah como Parmar tienen, como se ha demostrado, la necesidad de denunciar la aún deficiente convivialidad multicultural y sexual.

\section{BIBLIOGRAFÍA}

"Afrocentric News". Disponible en: http:// www.afrocentricnews.com/html/cinema.html. Consulta 14/10/11.

"Answers.com. Ngozi Onwurah". Disponible en: http://www.answers.com/topic/ ngozi-onwurah. Consulta: 13/10/11.

"Escuela abierta de feminismo. Awa Thiam": Disponible en: $h t t p: / / w w w . e s c u e l a d e-$ feminismo.org/spip.php?article63. Consulta: 12/10/2011.

"Escuela abierta de feminismo. Pratibha Parmar": Disponible en: http:// www.escueladefeminismo.org/spip. php?article62. Consulta: 8/04/2011.

Dong, Lan (2004): "Gendered Home and Space for the Diaspora: Gish Jen's Typical American", Thirdspace 4 (1), 66-86.

Ferguson, Robert (1998): Representing 'Race.' Ideology, Identity and the Media, London, Arnold.

Foster, Gwendoline Audrey (1997): Women Filmmakers and the African and Asian Diaspora. Decolonizing the Gaze, Locating Subjectivity, Illinois, Southern Illinois University Press.

Recibido: 30 de abril de 2011

Aceptado: 30 de mayo de 2011
Gilroy, Paul (2005): Postcolonial Melancholia, New York, Columbia University Press.

Grewal, Shabnam et al. eds. (1988): Charting the Journey: Writings by Black and Third World Women, London, Sheba Feminist Publishers.

Hall, Stuart (1992): "The West and the Rest: Discourses and Power", en Formations of Modernity, ed. Stuart Hall y Bram Gieben, Cambridge, Polity Press, 275332.

Shohat, Ella y Robert Stam (1994): Unthinking Eurocentrism. Multiculturalism and the Media, New York, Routledge.

Walker, Alice (1982): The Color Purple, New York, Pocket Books.

Walker, Alice y Pratibha Parmar (1996): Warrior Marks: Female Genital Mutilation and the Sexual Blinding of Women, Florida, Harvest.

\section{FILMOGRAFÍA}

And Still I Rise (Dir. Ngozi Onwurah, 1993). A Place of Rage (Dir. Pratibha Parmar, 1991). Bhangra Jig (Dir. Pratibha Parmar, 1990 TV).

Brimful of Asia (Dir. Pratibha Parmar, 1988 TV). 
Coffee Coloured Children (Dir. Ngozi Onwurah, 1988).

Como agua para chocolate (Dir. Alfonso Arau, 1992).

Doctors (Serie televisiva, BBC - varios episodios dirigidos por Patribha Parmar).

Double the Trouble, Twice the Fun (Dir. Pratibha Parmar, 1992 TV).

Emergence (Dir. Pratibha Parmar, 1986).

Flesh and Paper (Dir. Pratibha Parmar, 1990). Fruits of Fear (Dir. Ngozi Onwurah, 1990).

Hang Time (Dir. Ngozi Onwurah).

Jodie: An Icon (Dir. Pratibha Parmar, 1996).
Khush (Dir. Pratibha Parmar, 1991).

Memsahib Rita (Dir. Pratibha Parmar, 1994, TV).

Monday's Girls (Dir. Ngozi Onwurah, 1993).

Nina's Heavenly Delights (Dir. Pratibha Parmar, 2006).

Playing Dead (Dir. Pratibha Parmar, 2008).

Sari Red (Dir. Pratibha Parmar, 1988).

Siren Spirits (Mini-serie Dir. Ngozi Onwurah y P. Parmar, entre otros, 1995, TV).

Sita Gita (Dir. Pratibha Parmar, 2000).

The Body Beautiful (Dir. Ngozi Onwurah, 1991).
The Colour of Britain (Dir. Pratibha Parmar, 1994, TV).

The Desired Number (Dir. Ngozi Onwurah, 1995).

The Righteous Babes (Dir. Pratibha Parmar, 1998).

Warrior Marks (Dir. Pratibha Parmar, 1993).

Wavelenghts (Dir. Pratibha Parmar, 1997).

Welcome II the Terrordome (Dir. Ngozi Onwurah, 1994).

White Men Are Cracking Up (Dir. Ngozi Onwurah, 1996).

Who Stole the Soul? (Dir. Ngozi Onwurah, 1992). 